Ann. Zootech., I972, 21 (I), 83-I05.

REVUE, BIBLIOGRAPHIQUE

\title{
LE TRANSIT DIGESTIF CHEZ LES MONOGASTRIQUES
}

\author{
I. - LES TECHNIQUES D'ÉTUdE \\ J.-P. LAPLACE \\ Station de Recherches sur l'Élevage des Porcs, \\ Centre national de Recherches zootechniques, I.N.R. A., \\ 78 - Jouy-en-Josas
}

\section{PLAN}

INTRODUCTION

Méthodes INDIRECTES

Temps de rétention dans le tube digestif.

Les substances de référence.

Mesure des temps de rétention.

Techniques radio-graphiques.

Substances radioopaques.

Radio-éléments.

Évaluation de l'activité propulsive.

Évaluation quantitative de la motricité gastro-intestinale.

Mécanogrammes.

Électromyogrammes.

Évaluation de l'activité propulsive.

Méthodes diRectes

Techniques volumétriques ou pondérales.

Technique des abattages.

Fistulation digestive.

Techniques débitmétriques.

Exploitation des données volumétriques.

Techniques d'aspiration et de dilution.

Repas d'épreuve en série.

Double échantillonnage.

Exploitation des données recueillies.

Discussion Ea Concrusions 


\section{IN'TRODUC'TION}

L'apport permanent de nutriments variés est une nécessité pour la compensation des dépenses de l'individu. Dans ce sens, le comportement alimentaire constitue un moyen primordial de l'homéostasie. Son indissociable complément est le phénomène de digestion défini comme l'ensemble de la transformation des matériaux ingérés, de l'assimilation des nutriments et de l'élimination résiduelle. Parmi les multiples processus mis en jeu, les mécanismes de transfert (transit digestif et excrétion) ont un rôle important. Cette fonction est assumée par la présence au niveau des viscères digestifs de mouvements complexes qui constituent la motricité du tractus gastro-intestinal.

Depuis près d'un siècle, cle nombreux expérimentateurs ont, à la suite de WEISKE (1897) et Marbaix (I 898), tenté de définir la vitesse de passage des aliments dans le tube digestif, et celle de l'élimination de leurs composants indigestibles. Ils se sont également attachés à la compréhension des modalités du transit au niveau des divers segments intestinaux. Ainsi que le rappellent Françors et al. (1968), on pouvait espérer établir une relation entre le temps de rétention des aliments et leur digestibilité, donnéc qui permettrait d'apporter une correction dans le calcul des bilans alimentaires.

Dans le cas du Porc, monogastrique majeur en raison cle son importance zootechnique, la connaissance des particularités du transit digestif, associées à telle ou telle modalité d'alimentation est particulièrement intéressante du fait de l'interdépendance entre le transit et la régulation du comportement alimentaire. Ainsi, les travaux de RÉrat et Lougnon (i963), d'Auffray et al. (1967), et de Laplace et Tomassone (I970) constituaient un premier pas dans ce sens, compte tenu des observations initiales de Leroy et Février (1947-1949). De façon analogue, la rapidité du transit digestif est un élément important de compréhension de la vitesse d'absorption des sucres (Aumaitre et al., 1969), ou des acides aminés - contenus dans les protéines, ou synthétiques apportés en supplémentation - (RÉRAT, 197I), ainsi que de l'état nutritionnel global. Il faut enfin souligner que cet état nutritionnel conditionne en partie l'état sanitaire de l'animal, notamment dans le cas des maladies chroniques ou subcliniques, dont l'importance est souvent méconnue (BraUde, 1967).

Avant de dresser un bilan de nos connaissances sur le transit digestif chez les monogastriques, il nous est apparu souhaitable de faire l'inventaire des nombreuses techniques à ce jour développées, et d'envisager les moyens nouveaux disponibles. Les techniques d'étude du transit digestif sont de deux ordres : d'une part, des méthodes indirectes d'appréciation du temps de rétention des ingesta ou de l'activité de la musculature gastro-intestinale; d'autre part, des méthodes directes de mesure du débit des matériaux alimentaires en un point déterminé du tube digestif, ou de la quantité de digesta présente à un instant donné dans tel compartiment digestif.

\section{I. - MÉTHODES INDIREC'TES}

\section{A. - Temps de rétention dans le tube digestif}

Le principe de cette technique est d'introduire dans le tube digestif des substances dites de référence, "marqueurs " solides ou hydrosolubles, ingérés par l'animal ou placés en un point quelconque du tube digestif par l'intermédiaire d'une fistule. Ces substances peuvent être, soit un élément constituant naturel de la ration, soit, plus souvent, un matériau étranger à la composition normale de l'aliment. 
Selon le mode d'administration et le site de récupération de la substance, on détermine :

- soit un temps de rétention global (ingestion-récupération dans les fèces) ;

- soit un temps de rétention partiel : (administration par intubation à un niveau quelconque - récupération par aspiration ou fistule, ou dans les fèces).

\section{Les substances de références.}

Les marqueurs les plus divers ont été utilisés par les expérimentateurs, ainsi que le rappellent FRANÇoIs et al. (1968) :

- Substances partiellement ou totalement indigestibles, naturellement présentes dans les aliments : lignine, cellulose, silice et chromogène, ou graines de céréales diverses. Ces marqueurs ont cependant été plus particulièrement utilisés chez les herbivores polygastriques.

- Marqueurs artificiels, au nombre desquels on peut citer :

- des particules de matière inerte : caoutchouc, polyéthylène, billes de verre, plastique (PAlohermo, I939; Alvarez et al., I924; KInG et al., r957 ; Johnson, I966), voire particules métalliques d'or, d'argent, de cuivre, ou d'acier (HoELzEL, I930) ;

- des matériaux colorés ou des colorants hydrosolubles tels que la paille colorée au vert brillant ou à la fuchsine basique, le rouge phénol (Mulrnos, I935; AbGarowicz (1), I948; Goldestern (1), I950 ; SMITH, I964);

- des substances chimiques indigestibles : oxyde de chrome, $\mathrm{Cr}_{2} \mathrm{O}_{3}$ (EDIN, I9I8-I926 in Hyden, I96I ; Paloheimo, I939; Schurch et al., I950; Haenlein et al., I966), oxyde ferrique (MANER et al., I962), chromate de soude $\mathrm{Na}_{2} \mathrm{CrO}_{4}$, polyéthylène glycol (HyDEN, I96I), résine complexe à l'or, dosé à $\operatorname{lo}^{-6} \mathrm{~g}$ par la technique dite "activation analysis" (MARTz et al., I969), utilisée très récemment aussi chez les ruminants avec l'or radioactif ${ }^{197}$ Au (MARTz et al., r971);

-- des composés radioactifs indigestibles : substances marquées au chrome radioactif ${ }^{51} \mathrm{Cr}$ (CAMERON, I960), qu'il s'agisse d'oxyde de chrome ou de chromate de soude, utilisables tous deux sous leur forme " marquée " (HANSkY et CONNEL., I962), or radioactif ${ }^{133} \mathrm{Au}$ (BRIs et al., I967), yttrium ${ }^{91} \mathrm{Y}$ (ABRAMs et BISHOP., I967) et terres rares radioactives (Marcus et al., r962; Huston et Elis., I965).

Parmi toutes ces substances, les plus fréquemment utilisées dans toutes les espèces sont le polyéthylène glycol (PEG) et l'oxyde de chrome $\left(\mathrm{Cr}_{2} \mathrm{O}_{3}\right)$. Cependant, la possibilité d'une perturbation de la digestion, l'existence de migrations différentielles des marqueurs par rapport à la phase liquide ou solide du contenu digestif, la complexité parfois du dosage de certains marqueurs, conduisent à ne retenir que quelques substances, comme étant actuellement les plus adéquates :

- le PEG, en qualité de marqueur de la phase liquide,

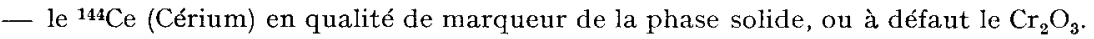

Les critères retenus pour la définition d'une substance de référence fiable sont:

- la détermination chimique précise de la substance, et un dosage aisé ;

- la non absorption ;

- la non production naturelle au niveau du tube digestif ;

- la non dégradation par les microorganismes;

- l'absence d'influence sur les divers processus;

— une vitesse de migration identique à celle de la phase étudiée du contenu intestinal.

II convient ici de souligner avec BLoom et al. (I967), le fait que la non absorption d'un marqueur doit être contrôlée par mesure de sa récupération dans des circonstances absolument identiques à celles de l'expérience.

(1) D'après FrançoIs et al. (Ig68). 
Le polyéthylène glycol, marqueur de la phase liquide.

L'utilisation du polyéthylène glycol a été particulièrement développée à la suite des travaux de Sperber et al. (1953) et de Hyden (I956). Il s'agit d'un composé polymérisé à longue chaîne, de formule générale $\mathrm{HO}-\mathrm{CH}_{2}\left(\mathrm{CH}_{2}-\mathrm{O}-\mathrm{CH}_{2}\right)_{n}-\mathrm{CH}_{2}-\mathrm{OH}$. Le PEG de poids moléculaire moyen 4 ooo ( $\mathrm{PEG}_{4}$ ooo) est le plus couramment utilisé. Sa distribution dans le contenu digestif du porcelet a été testée par MANNERs et al. (1967).

En effet, l'addition in vitro de solutions renfermant pour $100 \mathrm{ml}: 25 \mathrm{~g}$ de glucose, $0,42 \mathrm{~g}$ de PEG 4 ooo et $3,4 \mathrm{~g}$ de fluorure de sodium (conservateur), à du contenu gastrique ou intestinal, montre que le PEG 4 ooo se comporte différemment : ajouté au surnageant obtenu par filtration et centrifugation du contenu digestif, le PEG se distribue uniformément. Tel n'est pas le cas lorsqu'on l'ajoute au contenu digestif brut. Remarquons aussi l'extrême variabilité des taux de récupération du PEG selon les auteurs et l'espèce animale. IshiKawa et al. (1968) obtiennent chez le Porc 9I,7 p. Ioo, Corbett et al. (I956-I958) 96 p. Ioo chez la Vache, Hyden (I966) 97 p. Ioo chez l'Homme. Récemment, Ishikawa et al. (I968) ont comparé chez le Porc les taux de récupération du PEG 4 ooo et du $\mathrm{Cr}_{2} \mathrm{O}_{3}$ au cours de périodes de 5 jours, avec addition à taux constant $\mathrm{du}$ marqueur dans la ration. Ils ont alors observé une récupération incomplète du PEG et cherché à en déterminer l'origine. Les explications envisagées par ces auteurs sont au nombre de quatre :

a) Dégradation. - Le PEG n'est pas dégradé in vivo ainsi qu'il a été démontré par IsHI KaWA (1965), et in vitro par Smith (1959) et Jacobson (1963).

b) Absorption. - Le PEG est susceptible d'être éliminé dans l'urine lorsqu'il est introduit dans le sang (Schaffer (1) et al., 1947). Bien qu'il soit aisément dosable dans les urines, on ne peut l'y détecter après administration orale chez l'Homme (SCHEDL et al, I 966 ; SchAFFER et al. I950,), le Rat (Schaffer et al., 1950), le Mouton et la Vache (Sperber et al., r953; Downes $e t$ al., 1964), ou le Porc (Ishikawa et al., 1968), ce qui démontre qu'il n'est pas absorbé par l'intestin. A noter, cependant, que la non absorption n'est valable généralement que pour un tube digestif sain. Si la muqueuse est malade, il existe une possibilité d'absorption macromoléculaire. Ainsi en est-il dans la colite ulcérative (Millar et Brooke, ig66). Au demeurant, Sh1elds et al. (r968) constatent une non absorption du PEG 4 ooo par le gros intestin, qu'il soit normal ou pathologique.

c) Adsorption à la paroi digestive. - Il est important de souligner, sur ce point, qu'une récupération totale ne serait possible que par rinçage de l'espace intervillositaire chez le Rat (ABERDEEN, I960 et DaHLQUist, I963).

d) Analyse du PEG. - La technique même du dosage a été décrite par Hyden (1955). Mais les auteurs envisagent ici la possibilité pour certaines substances contenues dans l'intestin grêle d'interférer dans la technique de dosage au point d'entraîner des erreurs appréciables (Sмmтн, I958). Un dernier problème reste inconnu : celui d'une éventuelle liaison du PEG à divers matériels biologiques.

Quoi qu'il en soit, le PEG est particulièrement soluble dans l'eau. Il se distribue très vraisemblablement dans la fraction liquide des aliments (CORBETt et al., 1958). Il n'est pas absorbé alors que tel est le cas pour un marqueur soluble comme le chrome-5 I éthylène diamine tétraacétique ( ${ }^{51} \mathrm{Cr}$ EDTA) testé par Downes et al. (1964). Il est également dépourvu d'effets secondaires tels que les effets toxiques observés pour le polyvinyl alcool lors d'un usage répété (Yamatani et al., I968). Il constitue, en somme, un bon marqueur de la phase liquide des contenus digestifs.

\section{Chrome et cérium, marqueurs de la phase solide.}

Balch et al. (1957) observent que la concentration du $\mathrm{Cr}_{2} \mathrm{O}_{3}$ diminue dans le contenu gastrique, à un rythme voisin de celui de l'évacuation de la matière sèche. KING et al. (1957) estiment que ce marqueur est adsorbé sur les particules alimentaires, ou qu'il se loge dans les interstices des aliments fibreux. Mais, il faut remarquer que la récupération $\mathrm{du}_{\mathrm{Cr}_{2}} \mathrm{O}_{3}$ varie entre les déféca-

(1) D'après Ishikawa et Furutchi (I968). 
tions au cours du nycthémère de 59,8 à I 34,8 p. Ioo dans le cas du Cheval (HaEnlein et al., ig66). Dans ce cas, 76 p. Ioo de la variation au cours de 24 heures peut être exprimé par une fonction polynomiale du sixième degré. Cette courbe d'excrétion est, du reste, mieux cxprimée par une fonction périodique sinusoïdale. L'importance de la variation d'un jour à l'autre est, par ailleurs, aussi grande qu'à l'intérieur d'une période de 24 heures. A noter, enfin, que le $\mathrm{Cr}_{2} \mathrm{O}_{3}$ comme le $\mathrm{Na}_{2} \mathrm{CrO}_{4}$ sont dépourvus d'effet propre sur le transit digestif (Whitby et LANG, I96o ; Roche $e t$ al., 1957). En ce qui concerne la technique de dosage, les problèmes de détermination des oxydes de chrome utilisés comme indicateurs dans les expériences de digestion, ont été très récemment examinés par Petry et Rapp (I97I). En définitive, l'utilisation du $\mathrm{Cr}_{2} \mathrm{O}_{3}$ nécessite des dosages relativement longs pour un marqueur dont la liaison à la matière sèche de l'aliment est certaine, mais néanmoins imparfaite. Pour cette dernière raison, et compte tenu des irrégularités observécs par HaEnlein et al. (1966), il est bon d'être prudent dans l'usage qui peut en être fait. Son utilisation est en tout cas exclue pour une étude différentielle du transit des divers constituants de la matière sèche.

Un meilleur marqueur de la phase solide des contenus digestifs est constitué par le cérium $\left({ }^{144} \mathrm{Ce}\right)$. Cette substance est un radionucléide de la famille des lanthanides, dont tout l'intérêt et les avantages comme marqueur fécal, ont été soulignés et testés par François et al. (I968). Ces auteurs, rappelant toutes les caractéristiques physiques de la substance, en justifient le choix par rapport à d'autres radio-éléments tels que l'yttrium, en raison de la période longue du ${ }^{\mathbf{1 4 4}} \mathrm{Ce}$, qui permet une expérimentation à la fois complète et prolongée. Ils confirment chez le Rat, la parfaite liaison entre le ${ }^{144}$ Ce et la phase solide des contenus digestifs (MiLLER et al., I967), l'absence d'une résorption digestive du cérium (Huston et al., I965), et citent les résultats de HamiL.TON (1947) qui chiffre la résorption à moins de 0,05 p. Ioo de la dose ingérée. Même utilisés à de fortes doses, les lanthanides sont parfaitement tolérés (CochkaN (1) et al., I950). Enfin, le dosage radiochimique du cérium est relativement facile, et la précision des résultats excellente.

\section{Mesure des temps de rétention.}

La solution la plus simple consiste à décrire la vitesse de passage du marqueur, en tenant compte de sa première apparition dans les fèces (ou au niveau de collecte), et de sa dernière détection. Ceci fournit, par rapport au temps, la mesure des vitesses maximale et minimale à laquelle les différentes portions du tube digestif sont capables d'assurer le déplacement des contenus. Cependant, la première et la dernière manifestation du marqueur ne portent que sur de très faibles quantités. Aussi est-il préférable de se baser sur le temps nécessaire à l'excrétion de 5 p. 100 et 95 p. Ioo de la substance de référence. Ces valeurs ont une fiabilité beaucoup plus grande.

Une autre solution consiste à décrire le mouvement à travers le tube digestif de la portion contenant la quantité maximale de la substance de référence. Cela est beaucoup plus difficile, car la quantité de marqueur présente dans un segment dépend des vitesses non uniformes de mouvement de l'ensemble des segments qui le précèdent, aussi bien que de la localisation des matériaux qui sont déjà passés avant. La difficulté a été tournée par la compartimentation du tube digestif (Sikov et al., I 969). Ces auteurs ayant cu pour cela recours à l'autoradiographie, nous l'envisagerons plus avant.

Un moyen terme est apporté par Castre et Castle (1956), consistant à chiffrer le temps nécessaire au transit de l'ensemble de la masse alimentaire marquée, comme l'intervalle de temps entre l'excrétion $5 \mathrm{p}$. roo et l'excrétion $95 \mathrm{p}$. roo. Enfin, le terme le mieux adapté pour toute analyse statistique, en tant que valeur unique rendant compte du rythme de passage moyen de la totalité du repas marqué, est le coefficient de rétention $R$, proposé par CastLe (I956). Ce terme permet de comparer, sur toute leur longueur, les courbes d'excrétion de type sigmoïde, obtenues en exprimant, par rapport au temps, le pourcentage recueilli aux divers intervalles de temps de la quantité totale de marqueur récupérée, considérée comme la valeur Ioo p. Ioo. Le terme R, propor-

(1) D'après Françors et al. (1968). 
tionnel à la surface située à gauche de la courbe d'excrétion est calculé à partir du graphique en ajoutant, entre 5 et 95 p. Ioo, les temps d'excrétion relevés à intervalles de ro p. Ioo et en divisant cette somme par io.

\section{B. - Techniques radiographiques}

Lcs techniques radiographiques font appel, soit à des substances opaques au rayonnement $\mathrm{X}$, soit à des substances émettrices d'un rayonnement propre. Dans l'ensemble, toutes ces techniques se prêtent aisément à l'enregistrement des clichés par les moyens cinématographiques ou télévisés (ENGE, 1967). Dans le cas d'usage de substances radioactives, une variante technique peut être utilisée après sacrifice de l'animal; il s'agit d'autoradiographie.

\section{Substances radio-opaques.}

L'usage des sels de baryum ou de bismuth est déjà relativement ancien. McSwiney et SPURELL (1933), remplissant l'estomac avec des marqueurs radio-opaques, enregistraient sa surface projetée. Il est cependant difficile de déduire le volume du contenu gastrique de tels films à 2 dimensions. Néanmoins, des radiogrammes de l'estomac contenant un repas opacifiant, fournissent des résultats reproductibles chez l'Homme (HAwkins et al., I953). Une amélioration est apportée par le calibrage des images radiographiques; les surfaces correspondant à divers volumes de repas opacifiant servent à évaluer, en termes de volume gastrique résiduel, les images successives obtenues au cours de l'évacuation d'un repas d'épreuve radio-opaque (Hunt et KNox, I968). Une autre difficulté réside dans le fait que les substances opacifiantes sont susceptibles de modifier le rythme de l'évacuation gastrique. Pour cela, Annegers e t Ivy (I947) utilisent un repas expérimental normal et n'emploient la substance radio-opaque que comme élément différentiel pour évaluer le volume résiduel du repas d'épreuve au bout d'un temps donné.

Ces techniques peuvent également être utilisées dans le cas de l'intestin; HEdBLom et CANNoN (I909) mesuraient, après un repas de pommes de terre et de bismuth, la longueur des masses de bismuth dans l'intestin et obtenaient des résultats reproductibles. Mais, ces longueurs constituent un mauvais indice puisqu'elles vont diminuant au fil de l'absorption du diluant. L'usage de la ciné-radiographie a néanmoins permis l'obtention de résultats importants chez le Porcelet recevant du sulfate de baryum sous forme de bouillie lactée à raison de I $\mathrm{g}$ pour $2 \mathrm{ml}$ de lait (KIDDER et al., I96I-I968). Mais, il ne s'agit plus ici que d'évaluer un temps de passage. Dans ce seul but la technique radiographique se révèle particulièrement précieuse, notamment chez l'Homme (Matrson et al., 1962). Son utilisation pour une évaluation quantitative reste discutable.

Dès lors que la technique est fiable pour mesurer une vitesse d'évacuation gastrique ou un temps de passage dans l'estomac, il convient de préciser les conditions d'administration de la substance radio-opaque. Les repas d'épreuve proposés sont très divers et fournissent des résultats variables selon le diluant utilisé (lait, eau, gruau d'avoine etc.). Les principales critiques formulées visent la consistance non physiologique du repas obtenu et l'administration, préalable ou consécutive au diluant, de la substance opacifiante. La première critique est levée par le repas proposé par Goodman et al. (1958) : "sandwich, jambon et bouillie barytée ". Plus récemment, Stord et al. (I969) ont défini le repas d'épreuve : "steak et baryum " au cours duquel une gorgée de baryte est absorbée entre chaque bouchée de viande.

Reprochant au baryum la difficulté d'une étude quantitative, Hinton (ig66) a proposé une intéressante variante consistant en l'ingestion de pellets de polythène imprégnés de baryum, de tailles diverses. Le déplacement dans le tube digestif de ces solides radio-opaques est aisément suivi par des radiographies sériées. Quant au comptage dans les fèces, il s'effectue, sans manipulation de fèces, par radiographie du sachet plastique scellé, contenant chaque émission. Le comptage des pellets excrétés s'effectue sur le cliché. 


\section{Radio-éléments.}

Les radio-éléments sont ici utilisés non plus en vue d'un dosage quantitatif dans les fèces ou les contenus digestifs, mais pour un comptage sur l'individu vivant, ou pour une étude approfondie de l'ensemble du tube digestif (TODA et TOBE, I967), à un instant donné. CRAMER (I959) utilise le strontium radioactif pour l'étude du transit dans l'intestin du Rat, en déterminant la quantité de matériel radioactif présente dans les portions successives de l'intestin. HANSKY et CoNNelz (I 962) étudient le transit intestinal chez l'Homme avec du chromate radioactif ${ }^{51} \mathrm{CrO}_{4} \mathrm{Na}_{2}$. Cette même substance a été utilisée chez le lat pour l'analyse simultanée de l'évacuation gastrique et de l'activité propulsive de l'intestin grêle. Cette technique fournit, lors de l'abattage, le graphique de l'ensemble de la distribution du marqueur radioactif dans le tractus gastro-intestinal. Des données quantitatives précises peuvent être retirées d'un tel enregistrement par simple analyse planimétrique (DERвLом et al, , 1966).

A la même époque, Griffith et al. (1966) ont développé une technique d'étude sur le vivant de l'évacuation gastrique, qui a recours à l'inclusion dans un petit déjeuner anglo-saxon typique $(550 \mathrm{ml})$, de chromate de sodium radioactif. La validité des scintigrammes pour l'obtention d'informations quantitatives est démontrée par l'auteur. L'utilisation des isotopes radioactifs du chrome reste, malgré la complexité de l'appareillage, fort répandue pour l'étude de l'évacuation gastrique (HARVEY et al., I970). Une technique analogue, visant également l'évacuation gastrique in vivo, a été développée par Bromster et al. (I966 et r968) sur une variante de la technique de LuNDH (1957). Dans ce cas, l'épreuve consiste en l'ingestion rapide d'un repas d'un volume de $290 \mathrm{ml}$, aussi proche que possible d'un repas normal, contenant de l'albumine sérique humaine marquée à l'iode I $3 \mathrm{I}\left({ }^{131} \mathrm{I}-\mathrm{HSA}\right)$ et de l'iode I 25 -polyvinyl pyrrolidone ( ${ }^{125} \mathrm{I}$-PVP). La radioactivité de ${ }^{131} \mathrm{I}$ peut être suivie par mesure externe du rayonnement gamma, au niveau de l'estomac. ${ }^{125}$ I-PVP, substance inerte, permet d'effectuer une correction (éliminant la sécrétion de ${ }^{131} \mathrm{I}$ par les glandes salivaires et l'estomac). Mais la mesure s'effectue sur des échantillons de contenu gastrique, ce qui cnlève à la méthode une partic de son avantage de méthode externe. Semblable utilisation des isotopes de l'iode a cependant été faite pour l'étude de la fonction gastrique par DavcEv et TADZER (1968). Un aménagement intéressant est proposé par Rosswick et al. (1966). Il ne nécessite aucune préparation particulière du sujet, en lui laissant toute liberté d'activité et d'ingestion. De petites capsules d'araldite chargées en opacifiant et contenant moins de Io $\mu \mathrm{Ci}$ de ${ }^{131} \mathrm{I}$ sont ingérées. Leur localisation au $\mathrm{cm}$ près est obtenue avec un compteur à scintillation portable, chez l'individu équipé d'une grille en perspex sur l'abdomen.

Très récemment enfin, Sıkov et al. (1969) ont développé chez le Rat l'usage de l'administration intra-gastrique d'une solution de ruthénium Io6 en équilibre avec son produit de dégradation le rhodium I06. La solution ${ }^{106} \mathrm{Ru}-{ }^{106} \mathrm{Rh}$, à $\mathrm{pH}$ 2, n'est pas absorbée. La détection sur l'ensemble du tube digestif se fait, non plus par passage sous un détecteur (DERBLom et al., r966), mais par autoradiographie.

\section{3. Évaluation de l'activité propulsive.}

Les résultats recueillis par les techniques radiographiques n'ont pas fait l'objet d'un traitement très poussé de l'information. Pat contre, les données obtenues avec les radio-éléments ont été plus exploitées. Allant au-delà de l'analyse planimétrique, Sikov et al. (1969) ont défini l'exploitation quantitative des résultats, et déterminent sur l'ensemble du tube digestif 7 compartiments (gastrique, 4 d'intestin grêle, cæcum et côlon). On considère séparément le rythme de sortie de chaque compartiment au cours des intervalles entre les sacrifices. Le rythme de passage est calculé comme le pourcentage d'activité qui, présentée à ce segment, est évacuée par lui dans l'unité de temps. La technique suppose évidemment que tous les facteurs qui influencent le temps de passage soient constants entre les abattages. L'exploitation mathématique permet de définir la vélocité (dérivée de la distance parcourue par rapport au temps), de l'avancée des premiers éléments radioactifs, et fournit un modèle simple : 
Vélocité $=a-b \log _{10} x$

où $x$ peut être la longueur d'intestin franchie ou le pourcentage franchi de la longueur totale à traverser. Les mêmes données permettent enfin l'analyse du passage de la masse radioactive à travers chaque segment. L'expression graphique de cette rétention, compartiment par compartiment, est particulièrement intéressante.

Les résultats obtenus chez le Rat, à l'aide du chromate de sodium radioactif, ont fait l'objet d'une exploitation encore plus poussée : Grevsten et al. (1967-1969) ont développé une technique d'analyse mathématique de l'activité propulsive gastro-intestinale ainsi évaluée. Obtenant une évacuation gastrique de type exponentiel, exprimée par $x=\mathrm{e}^{k t}$, où $x$ est le volume gastrique, et où le volume évacué par unité de temps est représenté par la relation $\frac{\mathrm{d} x}{\mathrm{~d} t}=k \cdot \mathrm{e}^{k t}$; la fraction évacuée, ou quotient est donc $\frac{\mathrm{d} x / \mathrm{d} t}{x} \mathrm{c}^{\prime}$ est-à-dire $\frac{k \cdot \mathrm{e}^{k t}}{\mathrm{e}^{k t}}$ soit $k$ une constante. En se fondant sur I'hypothèse que la propagation dans l'intestin grêle répond au même principe, on définit un quotient propulsif $\mu_{i}$ du type :

$$
\mu_{i}=\frac{x_{i}}{\mathrm{~V}+\sum_{j=1}^{i-1} \mathrm{x}_{j}}
$$

où $\mu_{i}=$ quotient propulsif pour un segment intestinal donné $i$

$x_{i}=$ contenu de la fraction $i$

$\mathrm{V}=$ contenu de l'estomac

$\sum_{j=1}^{i-1} x_{j}=$ contenu de tous les segments situés en amont de la fraction $i$

Les auteurs démontrent alors que, dans des conditions normales, l'évacuation gastrique et la propulsion intestinale sont identiques pour les trois quarts environ de l'intestin grêle. La constante $k$ de la fonction exponentielle d'évacuation gastrique est identique au quotient propulsif $\mu i$.

\section{C. --Évaluation quantitative de la motricité gastro-intestinale}

Alors que les techniques radiographiques permettent une évaluation quantitative de l'activité propulsive intestinale, par l'image du contenu digestif opacifié en déplacement, il s'agit ici d'évaluer l'activité motrice du tube digestif. Celle-ci est supposée liée à la fois à la quantité de digesta et à leur vitesse de déplacement, puisqu'elle est la cause du déplacement plus ou moins rapide de volumes alimentaires plus ou moins importants. Ceci revient à apprécier lo facteur moteur du transit, et non plus l'effet transit lui-même.

\section{Mécanogrammes.}

La première solution, historique (LeGros et al., I 869 ; BAYLiss et STARLING., I 899), consiste en l'utilisation de ballonnets remplis d'eau, conjugués à un manomètre à eau et reliés à un tambour de Marey ou à un électro manomètre. Cependant, un tel procédé ne permet guère d'obtenir qu'une résultante entre la pression endo luminale réelle et la pression pariétale. Ils sont, de plus, sujets à des déplacements et susceptibles de provoquer, par leur seule présence, une motricité réflexe. Aussi cette technique a-t-elle été sévèrement critiquée par Quigley et Brody (1952). Une technique plus satisfaisante est celle dite des cathéters ouverts, qui offre une finesse de localisation bien supérieure et permet l'enregistrement d'une pression endo cavitaive ou endo luminale réelle, même pour des phénomènes très rapides. Leur mise en place sous anesthésie générale est réalisée selon la technique de WiTzel ou de Coffey. Chez l'Homme, des faisceaux de cathéters solidaires, dont les ouvertures digestives sont espacées de $7 \mathrm{~cm}$, peuvent être introduits par intubation, l'ensemble étant lesté d'un microballonnet de mercure (Friedman et al., I964). Quel que soit le mode 
utilisé, ces cathéters sont généralement employés en système hydraulique qui assure l'incompressibilité de la transmission. Une variante séduisante de l'enregistrement des pressions endo luminales consiste en l'ingestion de capsules baro-sensibles émettrices (FARRAR et al., I957-1958; Connell et Rowlands, 1960; Smith et Ridgway, ig62; Barany et Jacobsen, 1964 ; Banke, I968; Misiewicz, 1968). A noter, enfin, l'appareillage original d'Engelhardt et al. (ig67) pour la mesure par induction, in situ, de la motricité intestinale. Néanmoins, cette dernière technique ne semble pas avoir eu de développement général. Quant aux techniques radiotélémétriques, elles ne paraissent guère adéquates pour une étude du transit digestif, puisqu'elles permettent seulement de capter l'activité mécanique d'un territoire sans cesse changcant. De plus, le rythme de déplacement du capteur lui-même est distinct de celui des ingesta.

La recherche d'une mesure d'une pression strictement pariétale a conduit à l'emploi d'autres procédés. L'un consiste en l'insertion dans l'épaisseur de la musculature viscérale de microballonnets constitués par l'extrémité occluse et dilatée à la chaleur d'un fin cathéter de polyéthylène rempli de liquide et relié à un électromanomètre. Catte technique utilisée chez le Porc par AufFray et al. (1967) s'avèrı d'une grande finesse. L'autre fait appel à des jauges de contrainte (JACOBY et al., 1963). Le principe de la technique consiste à enregistrer la modification de la résistance d'un fil conducteur par suite de sa déformation. L'usage de ces jauges de contrainte permet d'envisager l'étude des phénomènes mécaniques par la mesure directe des déformations de la paroi des viscères. La motricité gastro-intestinale peut ainsi être enregistrée dans des conditions d'isométrie, et la force contractile exprimée en $\mathrm{g} / \mathrm{cm}^{2}$. La technique permet également entre autres avantages, longuement développés par ReinKe et al. (I967) et Rosenbaum et al. (1967), de séparer, dans d'excellentes conditions, l'activité des couches circulaire et longitudinale de l'intestin.

\section{Electromyogrammes.}

L'enregistrement de l'activité électrique du muscle gastro-intestinal, depuis Alvarez et MahoNEY (I922) et BozLer (1945 et 1946), est devenue l'une des techniques les plus élaborées dans l'étude de la motricité digestive. De très nombreux travaux (BAss et al., 1961 $a, b, 1965$; Daniel et al., 1960 $a, b, 1963,1966$; BoRToFf et $a l$., I965 $a, b, \mathrm{I} 967$ ) ont précisé les caractéristiques essentielles de cette activité viscérale. L'essentiel des connaissances a été rassemblé dans les remarquables revues de Daniel et IRwin (I968) pour l'estomac et de Bass (I968) pour l'intestin. Cette activité se compose d'une part, d'une activité périodique (ondes lentes) ou rythme électrique de base, résultant d'une dépolarisation rythmique des cellules de la musculeuse longitudinale, d'autre part, d'une activité rapide (potentiels de pointe) coordonnée par la précédente, en étroit rapport temporel avec elle et ayant pour origine la couche musculaire circulaire. Cette activité rapide est, à l'opposé du rythme de base qui n'est associé à aucun phénomène moteur, la source de l'activité motrice gastrointestinale. L'amplitude et la durée des bouffées de potentiels de pointe sont rigoureusement proportionnelles à la force et à la durée des contractions. L'activité propulsive, seule intéressante vis-à-vis du transit digestif, est le résultat d'une activité coordonnée des deux tuniques musculaires. Elle se caractérise par des potentiels de pointe de forte amplitude (Gonelta, I966, I $967 a$ et $b$ ) qui surchargent l'onde lente, elle-même associée à des potentiels d'amplitude très réduite.

En définitive, l'étroite relation de cause à effet entre activité électrique et activité propulsive fait de l'électromyographie digestive une technique de choix pour l'exploration du péristaltisme et de sa résultante, le transit des ingesta.

La dérivation de l'activité électrique sur l'animal éveillé, et plus encore chez l'Homme (Krapivin et Chernin, 1967 ; Netesin et Sha.poshnikov, i968), exclut l'usage de la dérivation bipolaire coaxiale au moyen d'aiguilles d'Adrian et Bronk, ou celle de microélectrodes en dérivation monopolaire. Schématiquement, certaines techniques fréquemment utilisées ont recours, soit à l'électroentérographie externe (DAvis et al., I957; CONDREA et al., I967), soit à un enregistrenent par électrodes maintenues au contact de la séreuse ou de la muqueuse. La possibilité de dériver au niveau de la paroi abdominale des potenticls en relation avec la motricité viscérale digestive 
(Thouvenot et al., I968) paraît riche de promesses en exploration fonctionnelle pour le clinicien, en dépit des difficultés d'interprétation. Ainsi, l'électrosplanchnographie est capable de déceler une lésion d'ulcération gastrique non décelable par d'autres méthodes (MarTin et al., I97I). L'inconstance des résultats de l'électrosplanchnographie, selon la disposition des électrodes, a conduit à la mise au point de l'électrogastroentérographie qui consiste à enregistrer au niveau des membres (4 électrodes) des variations lentes de potentiel, images de l'activité motrice digestive (Martin et Thillier, I97I). Les auteurs mentionnent une grande pureté des tracés qui intègrent toutes les activités. Cette technique simple à mettre en wuvre pormettra peut-être l'étude du transit digestif dans les conditions de la clinique.

La méthode de dérivation transmuqueuse, intra-intestinale (en dérivation généralement bipolaire), préconisée par GARRETT et al. (I963), procède toujours d'une technique d'intubation. L'usage d'une sonde à ballonnet appliquant les électrodes contre la muqueuse (TsUCHIDA et al., I966) est exclu du fait de l'occlusion résultant de la distension du ballonnet. Une solution adéquate est fournie par une sonde porteuse d'une cupule dans laquelle se trouvent placées les électrodes, et où peut être créée une dépression (roo à $200 \mathrm{~mm} \mathrm{Hg}$ ) ; le vide créé dans la cupule maintient les électrodes (argent, chrome ou acier) au contact de la muqueuse (Shiratori et al., i 969 ; Monges et al., $1970 a$ ). Cette technique, dite de l'électrode de succion, est utilisable à divers niveaux digestifs estomac ou intestin grêle (Monges et al., 1969, 1970 $b$ ). La méthode de dérivation transséreuse (mono ou bipolaire), évidemment réservée à l'animal, fait appel à la fixation, au contact de la paroi digestive, d'éléments porteurs des électrodes de dérivation : fil de platine pur isolé avec un élastomère de silicone formant plaquette suturée à la paroi intestinale (McCoy et BAKER, I968), ou bien plaquettes de téflon porteuses d'une ou deux électrodes de platine distantes de $5 \mathrm{~mm}$ (SzurszEWSKr et al., 1970).

Par rapport à ces dérivations tangentielles, la mise en place d'électrodes par piqûres à l'intérieur même de la paroi intestinale constitue une nette amélioration. Initialement utilisée sous la forme d'aiguilles électrodes implantées extemporanément au niveau des viscères préalablement extériorisés en préparation chronique (Amstrong et al., I956 ; Milton et al., 1956 ; Cartson et al., I966), cette technique fut largement perfectionnée pour permettre des enregistrements multiples de longue durée, sur l'Homme ou l'animal anesthésié (DANIEL et al., 1959): un fil d'argent (0,006 à o,or 8 inch) est glissé par ponction dans la paroi intestinale, juste sous la séreuse, et replié en forme de boucle orientée dans un plan perpendiculaire à la paroi intestinale. Une technique similaire développée par BUNKer et al. (1967) a permis enfin la dérivation chronique de l'activité électrique chez l'animal éveillé par l'usage de fil acier inoxydable enrobé de téflon, implanté dans la paroi de l'intestin grêle, et maintenu en place par des points de soie. Une technique similaire a été ultérieurement exploitée par Ruckebusch et al. (I968). Cette technique d'implantation chronique d'électrodes dans la musculeuse intestinale est particulièrement intéressante chez l'animal.

\section{3. Évaluation de l'activité propulsive.}

Les divers problèmes méthodologiques d'interprétation ou d'analyse des enregistrements de l'activité digestive ont déjà été discutés (BESAnçon, I967 ; ConnELL, I968). Très schématiquequement, le problème de l'évaluation du transit à partir de la motricité digestive nécessite de fournir pour chaque intervalle de temps consécutif un chiffre global exprimant les composantes de l'activité motrice et refétant par là le niveau d'activité du secteur digestif concerné. Une méthodologie relativement simple a été développée pour les mécanogrammes par Deller et WANGEL (1965). Ces auteurs ont proposé un index de motricité, exploité par eux sur les enregistrements de pression obtenus par la technique dite des cathéters ouverts. Il est défini par la formule $\frac{\sum(\mathrm{A} \times \mathrm{D})}{\mathrm{T} \times \mathrm{IO}}$ où A est l'amplitude de chaque onde de pression en $\mathrm{mm}$ de mercure, $\mathrm{D}$, la durée de l'onde en secondes, $\mathrm{T}$ la durée de l'intervalle de temps sur lequel portent les mesures. Cet index exprime la sommation des ondes successives au cours de cet intervalle de temps. Mrsiewicz (I968) a apporté un très important perfectionnement en introduisant l'analyse 
sur ordinateur qui fournit pour chaque unité de temps : le nombre d'ondes de pression par minute, la fréquence d'apparition d'ondes appartenant à chacune des ro classes de pression en $\mathrm{cm}$ d'eau représentant l'intervalle o à $100 \mathrm{~cm}$ d'eau et la fréquence d'apparition d'ondes appartenant à chacune des 6 classes de durée (en secondes) définies par l'auteur. L'exploitation de cette masse de données reste aisée grâce au calcul sur ordinateur et fournit un moyen d'une extrême puissance. Les deux solutions ci-dessus rapportées sont directement transposables dans le cas d'emploi de jauges de contrainte, où la pression est simplement exprimée par une force développée en grammes. Dans un cas comme dans l'autre, on dispose donc d'un reflet chiffré précis de l'intensité du travail mécanique de l'intestin pour le site considéré.

En ce qui concerne les techniques électromyographiques, le problème paraît, au premier examen, plus complexe. Il suffit en fait, soit de transformer les signaux par intégration automatique, soit de procéder à une analyse statistique de l'amplitude des signaux. Dans le premier cas, l'électromyogramme peut être transformé en son enveloppe redressée dont le tracé est analysable à l'instar d'une courbe d'enregistrement de pression. Il peut aussi être intégré de façon à fournir la sommation de l'activité musculaire au cours d'un intervalle de temps choisi. Dans le second cas, l'adaptation à l'électromyographie, de l'analyseur statistique d'amplitudes utilisé en électroencéphalographie quantitative (Drоноскт, 1967) peut être envisagée, ce qui revient à fournir, pour chaque intervalle de temps, le spectre de distribution des amplitudes prédéfinies, au cours de l'intervalle de temps considéré. Le schéma obtenu est alors identique à celui de l'analyse des pressions de Misiewicz (Ig68). Une telle possibilité, que nous avons très récemment envisagée (LAPLACE, I97I), présenterait par rapport au simple comptage de la présence ou de l'absence des bouffécs de potentiels de pointe, l'avantage de tenir compte du nombre et de l'amplitude des potentiels. Si cette adaptation s'avère réalisable, il semblerait que l'on dispose, par là, de la méthode la plus précise de mesure de l'importance numérique et de l'intensité des activités motrices digestives.

Loin d'être utopique, cette méthode d'étude semble avoir trouvé une consécration dans le remarquable appareillage élaboré par JELL ( I970). L'unité de contrôle à 8 voies, analyseuse et rythmeuse, mise au point par cet auteur, permet une conversion séquentielle, en formes digitales (avec impression ou perforation sur rubans pour unités d'entrée d'ordinateur) de données analogiques mixtes telles que variations lentes ( $\mathrm{EEG}^{*}$, température) ou impulsions (potentiels d'action). Elle peut, cn outre, assurer divers comptages : totalisation, intervalles, périodes. La mise en œuvre de cette technique apporterait probablement beaucoup à l'étude de la fonction digestive grâce à la possibilité d'enregistrement simultané en 8 sites du tube digestif, et à celle d'une analyse à la fois précise, complète et aisée pour le traitement de l'information.

\section{II. - MÉTHODES DIRECTES}

Par rapport aux méthodes indirectes, visant l'obtention d'un temps de rétention ou d'un reflet du transit, soit dans ses effets (répartition d'une substance radio-opaque ou de radio-éléments), soit dans sa causalité (motricité digestive), les méthodes directes ont pour but la mesure de la quantité de digesta présente, à un instant donné, dans un compartiment digestif, et de leur rythme de sortie de ce compartiment. La mesure de quantité peut être exprimée, soit par un volume de contenu digestif brut, soit par un poids de matière sèche. Le problème est compliqué, d'une part, du fait de la dilution du volume initial par les sécrétions digestives, d'autre part, en raison de la réduction de la quantité de matière sèche par suite de l'absorption digestive, et de la résorption d'eau.

(*) E E G. = Électro-encéphalographie. 


\section{A. - Techniques volumétriques ou pondérales}

\section{Techniques des abattages.}

Le principe đe l'abattage des animaux permet de procéder, quantitativement et séparément, au prélèvement des contenus alimentaires des divers segments du tube digestif. L'abattage des divers groupes d'animaux à des intervalles de temps différents après les repas expérimentaux permet d'obtenir un tableau du transit digestif pour une espèce animale et un aliment donné. L'écueil essentiel de la technique tient à l'impossibilité inhérente de suivre l'évolution du transit chez un même individu ce qui a pour fréquente conséquence l'obtention d'une forte variabilité individuelle. Cette technique, d'un usage très répandu pour une petite espèce telle que le Rat (Rosenthal et Nasset, I958; Rozen, r967), a pu être utiliséo également chez le Porc (Rérat et Lougnon, 1963). Il est alors possible de déterminer les quantités de matière sèche, ou de chacun des constituants de la matière sèche au niveau des divers segments du tube digestif, ce qui n'est pas toutefois l'apanage exclusif de cette technique.

\section{Fistulation digestive.}

Les techniques de fistulation digestive ne sont cortes pas nouvelles (MANn et BolLMAN, I93I). La technique de fistulation dite réentrante constitue un moyen expérimental précieux, mais assez peu utilisé pour les diverses espèces animales monogastriques à l'exception du Porc. Par ailleurs, chez l'Homm?, diverses études sur le transit digestif ont pu être conduites sur des sujets porteurs de fistules digestives traumatiques ou pathologiques. Dans le cas du Porc, la technique de fistulation réentrante a été introduite sur l'exemple des techniques développées chez les ruminants à la suite des travaux de Ash (1962). La technique de Ash transposée au Porc a ainsi été utilisée au niveau de l'iléon par Cunningham et al. (I963), à divers niveaux du jéjunum par Noakes et al. (1967) et sur le duodénum par Auffray et al. (1967). Dans son principe, cette technique consiste à interrompre la continuité digestive, à mettre en place une canule dans chacun des abouts intestinaux, et à relier les deux canules par un pontage extérieur. Ceci permet, après ouverture, de collecter les effluents à la canule proximale, et de les réinjecter après mesure - voire échantillonnage - dans la canule distale. Au demeurant, la technique de fistulation simple reste en vigueur, notamment pour l'estomac (ABEL et Buck, 1967), le cæacum (Redman et al., 1964), voire le côlon hélicoïdal du Porc (LAPLACE, I970, non publié).

En ce qui concerne l'intestin grêle, les auteurs précédemment cités ont eu recours à des canules en chlorure de polyvinyle, mises en place par voie abdominale səlon le procédé classique de Crocker et Markowitz (Markowitz et al., i964). Cependant, la briéveté du duoclénum du Porc et son enfouissement sous le volet costal, ont conduit dans le cas particulier de ce segment intestinal, à rechercher une voie d'accès plus antérieure au niveau des dernières côtes préalablement reséquées (TKACHEV, I969; LAPLACE et al., 1970). L'observation pour des conditions expérimentales voisines, de résultats différents entre les travaux de AUfFRAY et al. (1967) et LAPLACE et al. (I970) - différence attribuée par ces derniers à la technique de fistulation - pose le problème de la validité de la fistulation comme méthode d'étude du transit digestif. Il reste toujours permis de se demander si les mécanismes physiologiques ne sont pas perturbés par la présence des canules. En dépit de l'élaboration par LAPLACE et al. (1970) d'un type de canules particulier, répondant à un maximum de conditions d'inocuité, il paraît souhaitable de corroborer les résultats recueillis par les techniques de fistulation chronique, par d'autres observations, radiographiques par exemple, chez l'animal intact.

Indépendamment de cet aspect matériel, la méthode de collecte et de réintroduction des effluents n'est pas indifférente. Ainsi, la dérivation du contenu duodénal, sans réintroduction, accélère l'évacuation gastrique (Тномas et al, 1934, chez lo Chien); une prompte distension duodénale par réintroduction massive des effluents, inhibe à l'inverse l'évacuation (SPurell, 1935 - chez le Chat ; Auffray et al., 1967 - chez le Porc). Il est donc impératif que la mesure à effectuer soit rapide, et que la réintroduction des effluents soit identique en volume et en rythme à leur 
émission au niveau de la canule proximale. Cette néccssité est relativement aisée à respecter lorsque seule est recherchée une mesura de volume du contenu intestinal apporté par chaque salve péristaltique. Il est beaucoup plus difficile de le réaliser lorsqu'on souhaite étudier le transit différentiel des divers constituants de la matière sèche. Cependant, il importe de respecter absolument le rythme d'émission lors de la réintroduction, car l'état physiologique de l'animal pout en souffrir.

La nécessité, pour une étude cinétique de la biochimie des contenus digestifs, de prélever pour chaque intervalle de temps un échantillon représentatif des effluents recueillis et réintroduits au cours de cette période, constitue une entrave sérieuse, eu égard à la régulation volumétrique précédemment mentionnée. Le problème de manipulation, pour l'échantillonnage régulier au cours d'intervalles de temps consécutifs très brefs, est résolu par la mécanisation des opérations de collecte, de mesure, prélèvement et réinjection. Un tel appareillage automatique a été récemment proposé par Axford et al. (I97I) et par ThIvend et Poncet (197 I), pour le Veau et le Mouton. L'utilisation d'un appareillage identiqus à celui de Thivend et Poncet (I97I) est actuellement envisagée à la Station dans le cas du Porc. Cependant, une telle solution a pour conséquence de substituer à la variable aléatoire de volume recueilli au cours d'un intervalle donné, une variable temps nécessaire au recueil d'un volume déterminé par le réglage du contacteur dans le récipient de collecte. Par ailleurs, le volume réinjecté est toujours inférieur au volume recueilli d'une quantité faible, mais constante, et l'appareillage introduit une nouvelle variable qui est le délai imposé entre collecte et réinjection, puisque le temps nécessaire au déclenchement est lui-même variable. A noter, cependant, que le volume correspondant au prélèvement pour dosages, peut être remplacé par un égal volume provenant d'un animal donneur.

En dépit de ces quelques critiques qui visent la valeur absolue des données volumétriques recueillies, il convient de reconnaître à cette technique son caractère unique en ce qu'elle permet l'étude du transit différentiel des divers nutriments de la ration. De surcroît, la fixité du mécanisme assure la reproductibilité des opérations, et toute comparaison des valeurs relatives du transit de divers aliments est donc possible.

\section{Techniques débitmétriques.}

L'utilisation d'un débitmètre in situ est évidemment exclue du fait de la géométrie essentiellement variable de l'intestin. Cette solution peut être envisagée au niveau d'une dérivation externe dans un tube à lumière fixe. Ainsi Grivel (I97I) a utilisé la débitmétrie à effet Doppler, avec une sonde intercalée entre les deux éléments d'une fistulation réentrante et une valve dans la lumière de la tubulure afin d'exclure les reflux de contenu duodénal. Une telle solution, a priori intéressante, n'est en fait acceptable que pour une étude sommaire, mais exclut toute mesure quantitative précise en raison de l'hétérogénéité des contenus digestifs. Au demeurant, semblable critique a été formulée par RuckeBusch et KAY (197I) qui reconnaissent, dans le cas des ruminants, la validité discutable de cette technique sur le plan quantitatif.

\section{Exploitation des données volumétriques.}

Dans la majorité des cas, les auteurs se sont bornés à présenter les résultats sous la forme de débits horaires moyens, minimaux et maximaux. Rérat et Lougnon (I963) ont recherché les relations entre les quantités de matière sèche, ou de certains de ses constituants, et le temps écoulé depuis le repas. Ils ont ainsi mis en évidence des relations de type exponentiel au niveau de l'estomac et de l'intestin. Il est probable qu'une exploitation plus poussée dans ce sens, portant sur des quantités de contenu digestif, pourrait conduire à une analyse analogue à celle développée plus haut pour le chromate de sodium radioactif (GREvSTEN et al., I967-1969).

Une analyse mathématique plus élaborée a été tentée par LAPLACE et Tomassone (I970) pour l'examen de l'évolution dans le temps du volume émis par l'estomac après un repas, la collecte s'effectuant au niveau du duodénum proximal. Le processus consiste à rechercher la 
fonction polynomiale répondant à une courbe qui passe au mieux par les valeurs observées et qui donne la description la plus proche de la réalité du phénomène. Pour chaque essai l'équation de régression polynomiale (Draper et SMITH, I966) de la variable expliquée $\mathrm{Y}$ (volume émis depuis le repas) est calculée en fonction des puissances successives de la variable explicative $x$ (temps). On recherche donc le meilleur ajustement de la courbe définie par les valeurs observées, et d'une courbe représentative simulant une fonction du temps définie par un polynôme du type $y=b_{0}+b_{1} x+b_{\mathbf{2}} x^{2}+b_{3} x^{3}$. Les auteurs procèdent ensuite à une analyse de variance (REES, Ig66) portant sur les coefficients de régression polynomiaux, afin de tester divers effets expérimentaux. Cette technique d'exploitation, nouvelle dans ce domaine, se présente comme particulièrement fine et d'une très grande puissance de discrimination.

\section{B. - Techniques d'aspiration et de dilution}

\section{Repas d'éprenve en série.}

Les techniques faisant appel à la dilution d'une substance de référence pour la mesure des volumes de contenus gastro-intestinaux, sont remarquables du fait de leur usage presque exclusivement dévolu à la clinique humaine, et de leur association à des techniques d'injection et de prélèvement par intubation naso-gastrique (EDLICH et al., I967). Le principe consiste donc à introduire de cette façon une solution dont le reliquat sera aspiré un certain temps après. Dans le cas de l'intestin grêle, l'intubation a recours à une sonde à lumières multiples, permettant l'injection en un point donné, et l'aspiration quelques centimètres, voire quelques mètres, plus en aval.

Cette technique a été principalement développée, et appliquée au cas de l'évacuation gastrique, par Hunt et SpurRell (I95I). Ces auteurs écartent en effet la solution, parfois utilisée, consistant à introduire dans l'estomac une quantité connue, puis au bout d'un temps $t_{1}$, à aspirer, mesurer et réinjecter le contenu gastrique résiduel et à renouveler l'opération à plusieurs reprises. Ils préfèrent administrer, à différentes occasions, une série de repas standards identiques, soit à divers sujets, soit à un même individu, à raison d'un seul essai par jour. Dans tous les cas, l'épreuve est interrompue après la première aspiration du volume résiducl. Le délai entre l'injection et l'aspiration variant d'un essai à l'autre, il est possible par la synthèse des données recueillies au cours d'une série de ces repas, d'obtenir un tableau complet de l'évacuation gastrique. Ceci n'est évidemment possible que pour des repas de caractéristiques assez définies pour permettre une bonne reproductibilité d'un jour à l'autre. Sont à éviter : toute réaction psychologique, nerveuse ou chimique de la part du sujet; toute substance susceptible d'action sur les phases psychique et chimique de la sécrétion gastrique, toutes conditions anormales de viscosité, pression osmotique et $\mathrm{pH}$. Le repas d'épreuve doit être aisé à injecter et à réaspirer, et doit contenir une substance facile à mesurer, non absorbée ou sécrétée. L'absorption d'eau étant négligeable par rapport au volume du repas (HUNT, I949 b), cette substance permet la mesure de la quantité du repas présente à un instant donné, sous réserve de l'erreur introduite par le volume des sécrétions salivaire et gastrique.

Le repas d'épreuve défini par Hunt et SpurRell (1951) est constitué par :

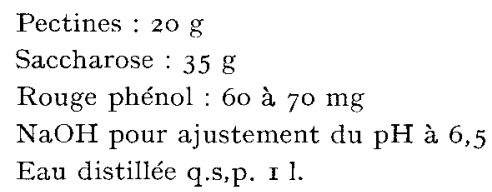

En effet, les pectines ne sont soumises à aucune dégradation dans l'estomac; le rouge phénol constitue un excellent index quantitatif du volume du repas; l'osmolarité obtenue fournit un point de congélation à $0,28^{\circ} \mathrm{C}$, ce qui correspond à une pression osmotique dont les effets sur l'évacuation gastrique sont faibles et à peu près constants; enfin, la viscosité à $37^{\circ} \mathrm{C}$ est de 37 à 43 centistokes, mais, en soi, la viscosité liée à la présence ou l'absence de pectines est incapable de modifier 
l'évacuation gastrique par rapport à un repas d'eau (HuNT, 1954). Il est à noter également que l'introduction directe du repas d'épreuve dans l'estomac n'entraîne aucune modification par rapport à son ingestion (HuNT et MACDONALD, I95I).

Après l'aspiration du volume gastrique résiduel, on procède à un ou deux lavages gastriques de façon à récupérer la totalité du rouge phénol. L'utilisation du procédé mis au point par Lipsky et al. (I969) peut être utile chez l'animal car il permet, par occlusion pylorique artificielle d'assurer une collecte parfaitement complète des substances résiduelles à l'instant choisi. Parallèlement à la mesure de concentration du rouge phénol, on peut par exemple procéder à la mesure de celle des ions $\mathrm{H}^{+}, \mathrm{Cl}^{-}$et de la pepsine, donc déterminer l'importance de la sécrétion gastrique. De même la transposition de la technique au niveau de l'intestin (BARREIRo et al, ig68) permet d'apprécier le transit digestif et l'absorption au cours du transit d'un repas d'épreuve. Une variante est proposée chez l'animal par l'association : abattages échelonnés et dilution du rouge phénol, pour la mesure simultanée de l'absorption et du transit gastro-intestinal chez le Rat (REynell et Spray, I956).

\section{Double échantillonnage.}

Ultérieurement a été proposée une variante améliorant la technique de HunT, encore que celle-ci soit toujours à juste titre en usage (CHANG et al., I968). Cette amélioration consiste en une technique dite de double échantillonnage (GEORGE, I968) basée sur le principe selon lequel le volume contenu dans un récipient peut être déterminé par l'augmentation de la concentration du marqucur dans le contenu, consécutivement à l'administration d'une nouvelle dose concentrée mais de faible volume, du mème marqueur. Le marqueur utilisé est toujours le rouge phénol, dosé ici au spectrophotomètre $(560 \mathrm{~m} \mu)$ pour accrôttre la précision par rapport au dosage colorimétrique pratiqué par HunT. Au bout d'un temps $t_{1}$ après l'administration du repas d'épreuve, on prélève une petite quantité du contenu gastrique, remplacée par une même quantité de marqueur concentré. Une minute plus tard, le brassage gastrique s'étant effectué, on prélève un nouvel échantilion. Si $V_{1}$ et $V_{2}$ sont respectivement le volume du contenu gastrique à déterminer et le volume de marqueur ajouté, $\mathrm{C}_{1}$ et $\mathrm{C}_{2}$ la concentration du rouge phénol dans les volumes $V_{1}$ et $V_{2}$, et $C_{3}$ la concentration finale obtenue dans le contenu gastrique, le volume $V_{1}$ recherché est $V_{1}=V_{2}\left(C_{2}-C_{3}\right)$. Cette manoeuvre est répétée à plusieurs reprises jusqu'à l'évacuation quasi complète de l'estomac, et en définitive la technique de double échantillonnage apporte une séquence d'informations pour un seul repas d'épreuve, ce qui constitue un net progrès par rapport à la technique de HunT.

\section{Exploitation des données recueillies par la technique d'aspiration-dilution.}

A l'instar de la fistulation digestive, et dans le cas le plus généralement envisagé de l'évacuation gastrique, les chiffres recueillis sont simplement rassemblés en fonction du temps. Pour Hunt et SpurRell (I95I), les points successifs exprimant l'évacuation gastrique en fonction du temps sont issus d'essais distincts. Pour GEORGE (I968) ces mêmes points peuvent appartenir à la même épreuve. Hors cette association graphique qui conduit la grande majorité des auteurs à concevoir l'évacuation gastrique d'un repas d'épreuve comme une fonction exponentielle (log volume/temps), on peut remarquer que ces techniques d'aspiration conduisent à apprécier le volume résiduel gastrique et non le volume évacué par l'estomac (cas de la fistulation duodénale haute).

\section{DISCUSSION E'T CONCLUSIONS}

Compte tenu des motivations préalablement développées, et au terme de cette revue exclusivement consacrée aux techniques d'étude du transit digestif, il convient de préciser l'objectif à atteindre, afin de mieux dégager l'utilité plus particulière de telle ou telle technique.

Annales de Zootechnie. - I972. 
Ainsi chez l'animal, une étude per se du transit digestif, visant à déterminer les temps de passage minimal et maximal, pourra avoir simplement recours à la mesure des temps de rétention. Cette technique n'apporte cependant que fort peu d'informations. Il est par conséquent bien préférable, étant libéré de toute autre contrainte expérimentale, d'analyser le passage de la masse alimentaire en fonction du temps. Dans ce cas, le choix de la technique de recueil res données volumétriques souhaitées sera conditionné par l'espèce animale en cause. Dès lors, les techniques de fistulation réentrante s'avèrent les plus intéressantes pour cles sujets tels que le Porc. Pour de petites espèces, par contre, pour lesquelles l'abattage ne constitue pas une charge financière, ou pour lesquelles ne se pose aucun problème particulier de contention, les techniques fondées sur l'emploi de substances radio-opaques et plus encore des radio-éléments (qui assurent une meihleure mesure quantitative) seront mieux adaptées. Là encore, queloues nuances doivent être apportées : ainsi l'autoradiographie est généralement limitée dans son application aux petites espèces à tube digestif court. Par ailleurs, les techniques radiographiques (opacifiants ou radioéléments) ne sont nullement inutilisables chez les grandles espèces et, le cas de l'Homme mis à part, on sait l'abondance et la qualité des résultats ainsi acquis chez le Chien. Mais cette solution n'est que difficilement envisageable chez le Porc, pour lequel une adaptation aussi parfaite que chez lc Chien ne saurait être obtenue, et dont la contention scrait un facteur de perturbation non négligeable. Par conséquent, les critères de choix sont tributaires - toutc disponibilité matérielle mise à part - de l'espèce animale, de ses capacités d'apprentissage ou de sa tolérance de la contention, et de la longueur de son tube digestif. A ces facteurs s'ajoutent le prix de revient de l'animal comme celui des radio-éléments par cxcmple, dont la quantité nécessaire variera sclon le volume du tube digestif et des ingesta qui y transitent.

Si l'on s'intéresse à la fois au transit différentiel des clivers constituants de la matière sèche et à la relation volume/temps, les techniques d'abattage ou de fistulation sont toutes indiquées. En effet, celles d'aspiration et de dilution sont véritablement l'apanage de la clinique humaine. Il convient cependant de remarquer l'imperfection inhérente aux deux techniques : pour l'une, possibilité de cléplacements plus ou moins importants de matériaux alimentaires entre l'instant de l'abattage, et celui de la pose de clamps délimitant des compartiments. l'our l'autre, perturbations quasi inévitables du rythme du transit. si minimisées qu'clles puissent être par le perfectionnement de l'appareillage lors de l'utilisation de la technique de fistulation.

A la clifférence d'une telle étude per se, l'examen du transit digestif en tant que facteur concomitant des phénomènes chimiques de la digestion et de l'absorption, doit se plier à diverses contraintes expérimentales. Le plus fréquemment, la mesure du transit digestif est associée à des études nutritionnelles ou à des études de digestibilité. Dans ce cas, et il s'agit ici principalement du Porc, on a recours à des substances de référence. Le PEG et l'oxyde de chrome sont alors les substances les plus utilisées dans la mesure des temps de rétention. Lorsque la mesure du transit digestif est associée à une étucle d'absorption, le choix est à nouveau fonction de l'espèce. Dans le cas du Porc, la mesure quantitative de l'absorption est désormais pratiquée au niveau de la veine porte. L'association de la technique de fistulation réentrante est donc possible. Par contre, pour une espèce telle que le Rat (ou l'Homme) l'absorption est généralement étuciée par la mesure des quantités de telle ou telle substance restant dans l'intestin grêle. Ce sont donc les techniques d'aspiration (ou d'abattage), et de dilution-concentration d'un indicateur coloré qui répondront à cette double nécessité expérimentale.

Parallèlement à l'éventail relativement large des possibilités techniques, ce qui permet une adaptation aux conditions expérimentales les plus variées, l'utilisation proposéc cles diverses techniques $\mathrm{d}^{\prime}$ enregistrement de la motricité gastro-intestinale apparaît comme une voie nouvelle, réservée toutefois à une étude libre de toute autre contrainte expérimentale. L'absence de possibilités technologiques adéquates, comme celle d'une méthodologie précise et efficace d'analyse pour de très grands nombres de données de ce type, ne sont pas étrangères à cette brèche clans l'éventail des techniques d'étude du transit digestif. Les moyens nouveaux, tel que l'analyse statistique séquentielle, ou l'usage de l'unité analyseuse et rythmeuse de JeLL (I968) ouvrent 
une perspective nouvelle. Si l'adaptation de ces procédlés à l'objectif visé se révèle correcte, il est permis d'espérer beaucoup de cette méthode indirecte, notamment en raison de la finesse d'analyse offerte par l'électro-myographic digestive. Nous voulons voir là une direction particulièrement intéressante pour une recherche de l'optimisation de la fonction digestive. Rappelons, en effet, le caractère plus ou moins héréditaire des particularités individuelles du transit, ct l'existence, montrée chez le Rat par Hoelzel dès I930, de relations entre certaines caractéristiques du transit cligestif, et la fertilité des femelles, la viabilité de leurs produits et le sex-ratio dans leurs portées. Il n'est donc pas interdit d'espérer de méthodes fort élaborées, les connaissances nécessaires à la définition des circonstances favorisant une fonction digestive optimale a fin de faire exprimer aux animaux le maximum de leurs potentialités.

En conclusion, il existe actuellement un éventail fort large de techniques d'étude du transit digestif. Il n'existe pas de technique uniformément idéale, mais cles facteurs de choix pour adapter la technique au but recherché en tenant compte des diverses particularités de l'espèce envisagée. Il est en fin permis d'espérer la rapide adjonction aux possibilités classiques, des techniques indiroctes dérivées de l'étude de la motricité digestive.

Reçu pour publication en octobre 1971.

\section{SUMMARY}

\section{MEASUREMEN'T OF THE GASTRO-INTESTINAL, TRANSIT IN MONOGASTRIC ANIMALS.}

\section{I. - DIFFERENT AVAILABLE TECHNIQUES}

The different techniques available for measuring the gastro-intestinal transit in monogastric animals are indicated in the present review. The description of these techniques represents the first part of a general report on the present knowledge concerning the passage rate of ingesta through the digestive tract of monogastric animals.

The techniques are divided into two groups :

I. Indirect methods for estimating the retention time of ingesta, or the gastro-intestinal muscular activity.

2. Direct methods for determining the flow rate of ingesta at a given point of the digestive tract or the amount of digesta present at a given moment and at a given point of the digcstive tract.

There are three different indirect methods :

I. Method for measuring the retention times. The author has checked all the natural or artificial reference substances employed. After having defined the suitable characteristics of a good reference substance, two types of tracers have been retained : polyethylene glycol as tracer of the liquid phase and chromium oxide or, even better, radioactive cerium as tracers of the solid phase of digesta.

2. The radiographic techniques include : utilization of X-ray opaque substances and of self-radiating substances. Estimation of the flow rate by means of these two methods is discussed.

3. Special attention is paid to the description of the methods used to study the mechanical and electrical activity of the digestive tract. An attempt is made to determine the different utilization possibilities of these techniques in the study of the gastro-intestinal transit. The quantification of such recording has been submitted to a prospective analysis.

The direct methods can be divided into two groups :

I. Techniques for volume and weight measurements based on slaughtering of the animals and on gastro-intestinal fistulation. Particular attention is payed to the mechanical treatment of the date and accurate mathematical utilization of the results.

2. The aspiration-dilution techniques mainly used in man (serial test meals and duplicate sampling).

The criteria determining the choice of a technique are discussed according to the accurate object of the experiment and taking into account an eventual association of the gastro-intestinal transit study with other types of studies. 


\section{RÉFÉRENCES BIBIIOGRAPHIQUES}

Abel M., Buck W. B., I967. A technique for gastric cannulation of swine. Cornell Vet., 57, 383-389.

Aberdeen V., Shepherd P. A., Simmons W. J., ig6o. Concurent measurement, in unanesthetized rats, of intestinal transport and fat absorption from the the humen. Quart. J. Exp. Physiol., 45, 265-274.

Abrams G. D., Bishop J. E., I967. Effect of the normal microbial fiora on gastro intestinal motility. Proc. Soc. Exp. Biol. Med., 126, 30г-304.

Alvarez W. C., Mahoney L. J., 1922. Action currents in stomach and intestine. Am. J. Physiol., 58, $476-493$.

Alvarez W. C., FREEDlander B. L., I924. The rate of progress of food residues through the bowel. J. Amer. Med. Ass., 83, 576-58o.

ANNEGERS J. H., IVy A. C., 1947. The effect of dietary fat upon gastric evacuation in normal subjects. Am. J. Physiol., 150, 46r-465.

Armstrong H. I. O., Milton G. W., Sмith A. W. M., i956. Electropotential changes of small intestine. J. Physiol. Londres 131, I47-I53.

Ash R. W., I962. Gastro-intestinal re-entrant canulae for studies of digestion in sheep. Anim. Prod., 4, 309-3I2.

Auffray P., Martinet J., Rérat A., 1967. Quelques aspects du transit gastro-intestinal chez le Porc. Ann. Biol. anim. Bioch. Biophys., 7, 261-279.

Aumaitre A., Février C., Rérat A., Rigaud J., Thivend P., I969. Application de l'analyse en continu à l'étude des variations de la glycémie du sang porte au cours de la digestion chez le Porc. C. R. Acad. Sci., $268 D, 7$ I 7-720.

AxFord R. F. E., Evans R. A., OFFER N. W., I97r. An automatic device for sampling digesta from the duodenum of the sheep. Res. Vet. Sci., 12, I28-1 3 I.

Balch C. C., Reid J.T., Stroud J. W., 1957. Factors influencing the rate of excretion of administered chromium sesquioxide by steers. Br. J. Nutr., 11, I84-197.

BANKE L., 1968. Radiotelemetry in the examination of intestinal peristalsis (danois). Ugeskr. Laeg., 130, $500-504$.

BARANY F., JACOBSEN B., r964. Endoradiosonde study of propulsion and pressure activity induced by test meals, prostigmine and diphenoxylate in the small intestine. Gut. 5, 90-95.

Barreiro M. A., McKenna R. D., Beck I. T., I968. Determination of transit time in the human jejunum by the single-injection indicator-dilution technic. Am. J. Dig. Dis., 13, 222-233.

Bass P., Code C. F., Lambert E. H., I96r $a$. Motor and electric activity of the duodenum. $A m$. J. Physiol., 201, 287-29I.

Bass P., Code C. F., Lambert E. H., r $96 \mathbf{r} b$. Electric activity of gastro duodenal junction. $A m$. J. Physiol. 201, 587-592.

BASS P., Wiley J. N., I965. Electrical and extra luminal contractile force activity of duodenum of the dog. Am.J.Dig. Dis., 10, 183-200.

Bass P., I968. In vivo electrical activity of the small bowel, pp. 205 I-2074 in Handbook of Physiology, sect. 6 Alimentary Canal., Vol. IV, Motility, Am. Physiol. Soc. Ed., Washington.

Bayliss M. W., Starling E. H., I 899. The movements and innervation of the small intestine. J. Physiol., Londres, 24, 99-I43.

Besançon F., I967. The current role of the graphic method in gastroenterology Acta Gastroent. Belg., 30, $704-707$.

Bloom D. S., Jacobson E. G., Grossman M. I., 1967. Validation of dilution indicators in the stomach. Gastroent., 52, 205-210.

BortofF A., WEg N., I965a. Transmission of electrical activity through the gastroduodenal junction. Am. J. Physiol., 208, 53I-536.

Bortofr A., I965 $b$. Electrical transmission of slow-waves from longitudinal to circular intestinal muscle. Am.J. Physiol., 209, I254-1260.

BortorF A., I967. Configuration of intestinal slow-waves obtained by monopolar recording techniques. Am. J. Physiol., 213, 157-162.

Bozler E., I945. The action potentials of the stomach. Am. J. Physiol, 144, 693-7oo.

BOzLER E., 1946. The relation of the action potentials to mechanical activity in intestinal muscle. Am. J. Physiol., 146, 496-501.

Braude R., 1967. The effect of changes in feeding patterns on the performance of pigs. Proc. Nutr. Soc., 26, I63-18I.

Bris F. J., Dyer I. A., Teare I. D., I967. Radiogold : a technique for measuring changes in rate of ingesta passage in the ruminant, as influenced by Diazepam. Agron. J., 59, 225-257. 
Bromster D., Carlberger G., Lundh G., I966. Measurement of gastric emptying rate. Lancet, 2, 224,225 .

Bromster D., Carlberger G., Lundh G., I968. Measurement of gastric emptying rate using ${ }^{131}$ I-HSA. A methodological study in man. Scand. J. Gastroent., 3, 64I-653.

Bunker C. E., Johnson L. P., Nelsen T. S., ig67. Chronic in situ studies of the electrical activity of the small intestine. Arch. Surg., 95, 259-268.

CAmeron A. D., I960. Gastro-intestinal blood loss measured by radioactive chromium. Gut. 1, I77-182.

Carlson H. C., Code C. F., Nelson R. A., I966. Motor action of the canine gastroduodenal junction: A cineradiographic, pressure and electric study. Ann.J. Dig. Dis., 11, I55-I72.

CASTLE Elizabeth J., I956. The rate of passage of foodstuffs through the alimentary tract of the goat. I. Studies on adult animals fed on hay and concentrates. Br. J. Nutr., 10, I5-23.

CASTLE Elizabeth J., CASTLE M. E., r956. The rate of passage of food through the alimentary tract of pigs. J. Agric. Sci., 47, x96-203.

Chang C. A., McKenna R. D., Beck I. T., I968. Gastric emptying rate of the water and fat phases of a mixed test meal in man. Gut. 9, 420-424.

Condrea H., Manoach M., Gitter S., Feller N., 1967. External electroenterography. Arch. Surg., 94, II 2-I I5.

Connell A. M., Rowlands E. N., I960. Wireless telemetering from the digestive tract. Gut. 1, 266-272.

Connell A. M., I968. Measurement of intra luminal pressures. Problems of methodology and interpretation, and analysis of records. Am.J. Dig. Dis., 13, 397-409.

Corbett J. L., Miller T. B., Clarke Eileen W., Florence E., I956. The use of polyethyleneglycol as an inert reference substance for the estimation of faecal output. Proc. Nutr. Soc., 15, V (abstr.).

Corbett J. L., Greenhalgh J. F. D., Gwyn P. E., Walker D., 1958, Excretion of chromium sesquioxide and polyethylene glycol by dairy cows. Br. J. Nutr., 12, 266-276.

Cramer C. F., I959. Movement of radiostrontium through intestinal tract of fed or fasted rats. Proc. Soc. Exp. Biol. Med., 102, 5II-5Iz.

Cunningham H. M., Friend D. W., Nicholson J. W. G., r963. Observations on digestion in the pig using a re-entrant intestinal fistula. Can. J. Anim. Sci.48, 215-225.

Dahlquist A., Thomson D. L., I963. The digestion and absorption of sucrose by the intact rat. $J$. Physiol., Londres 167, I93-209.

Daniel E. E., Carlow D. R., Wachter B. T., Sutherland W. H., Bogoch A., i959. Electrical activity of the small intestine. Gastroent., 37, 268-28I.

Daniel, E. E., Honour A. J., Bogocir A., r960 a. Electrical activity of the longitudinal muscle of dog small intestine studied in vivo using micro electrodes. Am.J. Physiol, 198, II3-II8.

Daniel E. E., Wachter B. T., Honour A. J., Bogoch A., rg6o $b$. The relationship between electrical and mechanical activity of the small intestine of dog and man. Can. J. Biochem. Physiol., 38, $777-790$.

Daniel E. E., Chapman K. M., I963. Electrical activity of the gastro-intestinal tract as an indication of mechanical activity. Am. J. Dig. Dis., 8, 54-102.

DANiEL E. E., WiebE G. E., I966. Transmission of reflexes arising on both sides of the gastro duodenal junction. Am. J. Physiol., 211, 634-642.

Danifl E. E., Irwin J., I968. Electrical activity of gastric-musculature, pp. I969-I984, in Handbook of Physiology Sect. 6, alimentary Canal, Vol. IV, motility, Am. Physiol. Soc. Ed., Washington.

Davcev P., TADzer I., rg68. Study of evaluation function of stomach by means of radioisotopes (croate). God. Zborn. Med. Fak. Skopje, 14, 103-I10.

Davis R. C., Garafolo L., Gault F. P., I957. An exploration of abdominal potentials. J. Comp. Physiol. Psychol., 50, 519-525.

Deller D. J., Wangel A. G., I965. Intestinal motility in man : A study combining the use of intraluminal pressure recording and cineradiography. Gastroent., 48, 45-57.

Derblom H., Johansson H., Nylander G., 1966. A simple method of recording quantitatively certain gastro-intestinal motility functions in the rat. Acta Chir. Scand., 132, I54-165.

Downes A. M., McDonald I. W.. r964. The chromium-5 I complex of ethylenediamine tetraacetic acid as soluble rumen marker. Br. J. Nutr., 18, I53-г62.

Draper N. R., Smith H., I966. Applied regression analysis. I vol. 487 p., John Wiley Ed., New York.

Droноскі Z., I967. L'analyseur statistique d'amplitudes de I' E E G Revue Neurol., 117, 482-484.

Edlich R. F., Gedgaudas E., Leonard A. S., Wangensteen O. H., ig67. New long intestinal tube for rapid non operative intubation. A preliminary report. Arch. Surg., 95, 443-449.

ENGE I., 1967. Cinematography of intestinal motility (norvégien). T. Norsk. Laegeforen, 87 (Suppl.), $887-888$.

Engeliardt W. V., Tolkmitt G., Ehrlein H. J., Stahlgreen L. H., I967. Ein einfaches Gerät zur induktiven Messung der Darmmotilität mit implantierten Spulen. Pflü. Archiv., 294, 229-234. 
Farirar J. T., Zworykin V. K., BaUm J., r957. Pressure-sensitive telemetering capsule for the study of gastro intestinal motility. Science, 126, $975-976$.

Farrar J. T., Bernstein J. S., I958. Recording of intra luminal gastro-intestinal pressures by a radiotelemetering capsule. Gastroent., 35, 603-6I 2 .

François E., Comperre R., Rondia G., r968. Étude comparée de la vitesse de passage des aliments et des résidus alimentaires non digérés dans le tractus digestif du Rat et du Mouton. Bull. Rech. Agric. Gembloux 3, 655-688.

Friedman G., Waye J. D., Weingarten L. A., Janowitz H. D., I964. The patterns of simultanoous intraluminal pressure changes in the human proximal small intestine. Gastroent., 47, 258-268.

Gakrett J. M., Schi,egel J. F., Hoffman H. N., 1963. Intraluminal detection of intestinal electrical activity. Fed. Proc., 22, 225 (abst. 389).

GEorge J. D., I968. New-clinical method for measuring the rate of gastric emptying: the double sampling test meal. Gut. 9, $237-242$.

Gonella J., Gignoux I lélene, r966. Étude simultanée de l'activité électrique des fibres musculaires lisses longitudinales et circulaires du duodénum de lapin in vitro. C. R. Acad. Sci., 262, 570-573.

Gonflea J., I967a. Étude de l'activité électrique des tuniques musculaires de l'intestin pendant les mouvements de segmentation et le péristaltisme. J. Physiol., Paris, 59, 4I6-4I7.

Gonella J., I967 b. Eitude électrophysiologique de la motricité intestinale. Thése Docteur Sci. Nat., Marseille.

Goodman E. N., Schlarger R., Harvey H. D., Colcher H., I958. Electrogastrographic and radiologic studies in patients with the dumping syndrome. Clin. Res., 6, 27 I (abstr.).

Grevsten S., Johansson H., Iindouist B., Nylander G., Ig69. Further analysis of a mathematical method of evaluating gastro intestinal propulsive motility. Acta Chir. Scand., 135, $237-240$.

Gressten S., Johansson H., Nrlander G., 1967. Analysis of a quantitative method of evaluation as applied to the propulsive gastro-intestinal motility in the rat. Acta Chir. Scand., 133, 563-567.

Grifith G. H., Owen G. M., Kirkman S., Shlibds R., ig66. Measurement of rate of gastric emptying using chromium-5I. Lancet, 1, I 244-1245.

Grivis, Maric-Iaure, I97. Itude électronyographique comparée de la notricité intestinale. Thèse Docteur, Sci. Nat., Toulouse.

Hafilein G. F. W., Smith R. C., Yoon Y. M., ig66. Determination of the fecal excretion rate of horses with chronic acid. J. anim. Sci, 25, I091-1095.

HanskY J., CoNnell A. M., I962. Measurement of gastrointestinal transit using radioactive chromium. Gut. 3, 187-188.

Harvey R. li, Mackie D. B., Brown N. J., Keeling D. H., Davies W. T., i97o. Measurement of wastric emptying tine with a gamma camera. Lancet, 1, I6-I7.

Hawkins G. K., Margolin S., Thompson J. J., I953. Gastric emptying time : comparative studies with placebos, prantal and banthine. Gastroent., 24, I93-199.

Hedilom C. A., Cannon W. B., I gog. Some conditions affecting the discharge of food from the stomach Am.J. Med. Sci., 138, 504-521.

Hinton J.M., ig66. A new method for measuring gut transit times. Gut, y, 7r8 (Abstr.).

HOEL, EL F., 1930. The rate of passage of inert materials through the digestive tract. Am.J.Physiol., 92, 466-497.

Htivi J. N., rg49a. A critical study of some aspects of gastric function in man. Ph. D. Thesis, London, Univ. of London.

HUNT J. N., I949 $b$. The simultaneous estimation of the absorption of water and sulphaguanidine from the stomach of man. J. Physiol., Londres, 109, I 34-141.

Hunt J. N., Spurrell W. R., I95I. The pattern of emptying of the human stomach. J. Physiol., Londres, 113. I 57 -I 68 .

Hunt J. N., Mac Donald I., I95I. The effect of intragastric instillation of a meal on gastric function in man. J. Physiol., Londres, 113, $185-188$.

Hunt J. N., I954. Viscosity of a test meal, its influence on gastric emptying and secretion. Lancet, 1, I 7 - 8 .

Hun' J. N., Krox M. T., I968. Regulation of gastric emptying, pp. I9I7-I935 in Handbook of Phy. siology Sect. 6 Alimentary Canal, IV Motility, I vol. Amer. Physiol. Soc. Ed., Washington.

Huston J. F., Ellis W. C., I965. Evaluation of ${ }^{144} \mathrm{Ce}$ as an indigestible marker. J.anim. Sci., 24, 888 (Abstr.).

HYDEN S., 195.5. A turbidimetric method for the determination of higher polyethylene glycol in biological materials. Ann. Roy. A gric. Coll. Sweden, 22, 139-145.

Hrines S., 19.56. The recovery of polyethylene glycol after passage through the digestive tract. Ann. Roy. A gric. Coll., Sweden, 22, $4 \mathrm{II}-424$.

Hydi: S., I96I. The use of references substances and the measurement of flow in the alimentary tract. in Migestive plyysiology and nutrition of the rmminant. Lewis D., ed., Butterworths, Londres, 35-47.

Ishikawa S., 1965. Degradation of polyethyleneglycol in the digestive tract. Agric. Biol. Chem., 29, I 73 - I 80 . 
IshikaWA S., FURUich Y., I968. Reliability of polyethylene glycol as an indicator for digestion studies with swine. Part 2 : Variation of polyethyleneglycol excretion and estimate of digestibility. Agr. Biol. Chem., 32, I086-1092.

JacoBy H. I., Bass P., BennetT D, R., I963. In vivo extraluminal contractile force transducer for gastro-intestinal muscle. J. Appl. Physiol., 18, 658-665.

Jacobson E. D., Bondy D. C., Broitman S. A., Fordtran J. S., Ig63. Validity of polyethyleneglycol in estimating water volume. Gastroent., 44, 76r-767.

JELL R. M., I970. Eight channel scanning and timing controller for physiological data acquisition. Med. Biol. Engng., 8, 383-388.

Johnson R. R., I966. Techniques and procedures for in vitro and in vivo rumen studies. J. anim. Sci., 25, $855-875$.

Kidder D. E., Manners M. J., I968. Passage of food in the young suckling pig. Proc. Nutr. Soc., 27,46 A.

Kidder D. E., Manners M. J., McCrea M. R., I96I. The passage of food through the alimentary tract of the piglet. Res. Vet. Sci., 2, 227-23I.

KING K. W., Moore W. C., I957. Density and size as factors affecting passage rate of ingesta in the bovine and human digestive tracts. J. Dairy Sci., 40,528-536.

Krapivin B. V., Chersin V. V., i 967 . Study of the motor function of the stomach by the electrogastrographic method in patients with peptic ulcer (russe). Sovet. Med., 30, 57-60.

Laplace J.-P., Tomassone R., I97o. Evvacuation gastro-duodénale chez le Porc. Fistulation chronique par voie thoracique extra-pleurale ; recherche d'une technique d'analyse mathématique de l'évacuation. Ann. Zootech., 19, 303-332.

LAPlace J.-P., I 97 I. Motricitégastro-intestinale chez le Porc : Étude descriptive par électromyographie et corrélations nutritionnelles. Rec. Méd. vét. (sous presse).

Legros, Onimus, i 869 . Recherches expérimentales sur les mouvements de l'intestin. J. Anat., Paris, 6, $37-66$.

Leroy A.-M., Fŕvrier R., I947. Influence du mode de distribution des repas sur l'utilisation de la nourriture pour le Porc. Ann, agron., 17, 765-770.

Leroy A. M., Févrikr R., i 949 . Influence du nombre des repas sur l'utilisation de la nourriture par le Porc. Ann. agron., 19, 605-6II.

Lipsky M., Brown M., Harrower H., Timayer W. R., r969. An intermitent pyloric occluder for obtaining complets gastric contents in dogs. J. Appl. Physiol., 27, 737-739.

Lundh G., 1957. Intestinal absorption after partial gastrectomy. Acta Chir. Scand., 113, 431-435.

Maner J. H., Pond W. G., Loosli J. K., Lowrey R. S., I962, Effect of isolated soybean protein and casein on the gastric $\mathrm{pH}$ and rate of passage of food residues in baby pigs. J. anim. Sci., 21, 49-52.

MANN F. C., Bollman J. L., I93I. A method for making a satisfactory fistula at any level of the gastrointestinal tract. Ann. Surg., 93, 794-797.

Manners M. J., Kidder D. E., r967. The distribution of polyethyleneglycol in gut contents. Proc. Nutr. Soc., 26, 25 (abstr.).

Marbaix O., I 898. I.e passage pylorique. Cellule, 14, 249-332.

Marcus C. S., Lengriman I. W., 1962. Use of radioyttrium to study food movement in the small intestine of the rat. J. Nutr., 76, I79-182.

Markowitz J., Archibald J., Downie H. G., 1964. Experimental surgery. $5^{\mathrm{e}}$ lid. I vol., p. 659 . Williams and Wilkins $C^{\circ}$, Ed. Baltimore.

Martin A., Thimider J. L., i97i. L'électro-gastroentérographie (E.GE.G.). Presse Méd., 79, I235-I237.

Martin A., Moliné J., Murat J., i97i. Apport de l'électrosplanchnographie (E.S.G.) au diagnostic préopératoire d'une ulcération gastrique. Presse Méd., 79, 1277-I278.

Martz F. A., Asay K. H., Wormington R. '., Leddicotte G. W., Daniels I.. B., Ig69. Activation analysis technique for measuring rate of passage. J.anim. Sci., 29 , I65 (abstr.).

Martz F. A., Asay K. H., Leddicotte G. W., Krause G., Daniels L. B., I97I, Activation analysis of gold-I97 for measuring rate of ingesta passage in ruminants. J. Dairy Sci., 54, 926-927.

Mattson O., Perman G., I962. Small intestine transit time studied in patients with the dumping syndrome. Acta Chir. Scand., 124, 326-332.

McCor E. J., Baker R. D., I968. Effect of feeding on electrical activity of dog's small intestine. Amer. J. Physiol., 214, I29I-I295.

McSwiney B. A., Spurrell W. R., I933. The outline method for investigating gastric motility. $J$. Physiol., Londres, 79, 423-436.

Militar D. M., Brooke B. N., Ig66. A method of serial portal venous sampling: its use in the demonstration of macromolecular absorption from the human colon. Gut, 7, 7I7 (abstr.).

Millis J. K., Perry S. C., Chandeer P. T., Cragle R. G., ig67. Evaluation of radiocerium as a non absorbed reference material for determining gastro-intestinal sites of nutrient absorption and excretion in cattle. J. Dairy Sci., 50,355-36r.

Murton G. W., Smitil A. W. M., Armstrong H. I. O., i 956. The electropotential changes in the duodenum of the dog. Bull. Soc. Int. Chirurg., 15, 29-35. 
Misiewicz J. J., 1968. Measurement of intraluminal pressures. Radiotelemetry, design of manometric studies, and computer analysis of records. Am.J. Dig. Dis., 13, 389-396.

Monges H., Salducci J., Roman C., I969. Étude électromyographique de la motricité gastrique chez l'Homme normal. Arch. Fr. Mal. App. Dig., 58, 5 I7-530.

Monges H., Salducci J., I $970 a$. A method of recording the gastric electrical activity in man. Amer. J. Dig. Dis., 15, $271-276$.

Monges H., Salducci J., I97o $b$. Étude électromyographique de la motricité duodènale chez l'Homme normal. Arch. Fr. Mal. App. Dig., 59, 19-28.

Mulinos M. G., I935. The value of selective drugs in the treatment of constipation. Rev. Gastroent., 2, 292-301.

Netesin G. V., Shaposhnikov I. G., I968. On a method of electrographic registration of the motor activity of the duodenum under experimental conditions (russe). Eksp. Khir. Anest., 13, 34-39.

Noakes D. E., Hill H. J., Freeman C. P., Annison E. F., ig67. The flow of intestinal digesta in the pig. Proc. Nutr. Soc., 26, VI (Abstr.).

Palonermo L., I939. Über die Verwendung des quantitativen Indikatorverfahrens bei Untersuchung der Nahrungsfortbewegung im Magendarmkenal. Tierernährung, 11, 370-388.

Petry H., Rapp W. I97I. Zur Problematik der Chromoxid-bestimmung in Verdauungsversuchen Z. Tierphysiol., Tievernähr., Futtermittelk., 2\%., 18I-189.

Quigley J. P., Brody D. A., I952. A physiologic and clinical consideration of pressures developed in digestive tract. Am. J. Med., 13, 73-8r.

Redman D. R., Teague H. S., Henderickx H. K., King N. B., I964. Cecal fistulation of the pig using two forms of indwelling cannulas. J. anim. Sci, 23, 1032-1035.

ReEs D. H., I966. The analysis of variance of designs with many non-orthogonal classifications. J.R. Statist. Soc. $B, 28$, IIO-II7.

Reinke D. A., Rosenbaum A. H., Bennett D. R., rg67. Patterns of dog gastro-intestinal contractile activity monitored in vivo with extraluminal force transducers. Am.J. Dig. Dis., 12, Ir3-I4I.

Rérat A., Lougnon J., I963. Études sur le transit digestif chez le Porc. Ann. Biol. anim. Bioch Biophys., 3, 21-27.

Rérat A., I97x. Mise au point d'une méthode quantitative d'ćtude de l'absorption chez le Porc. Journées d'Êtudes sur la Physiologie et la Biochimie de la digestion. Ann. Biol. anim. Bioch. Biophys., 11, 277-279.

Reynell P. C., Spray G. H., 1956. The simultaneous measurement of absorption and transit in the gastro-intestinal tract of the Rat. J. Physiol., Londres, 131, 452-462.

Roche M., Perez-Gimenez M. E., LAyrisse M., Diprisco E., I957. Study of urinary and fecal excretion of radioactive chromium ${ }^{51} \mathrm{Cr}$ in man. Its use in the measurement of intestinal blood loss associated with hookworm infection. J. Clin. Invest., 36, I 183 -1 92.

Rosenbaum A. H., Reinke D. A., Bennetr D. R., i 967 . In vivo force, frequency and velocity of dog gastro-intestinal contractile activity. Am.J. Dig. Dis., 12, I42-I53.

Rosenthal S., Nasset E. S., I958. Gastric emptying and intestinal absorption of carbohydrate and protein as infuenced by the nature of the test meal. J. Nutr., 66, 9I-IO3.

Rosswick R. P., STEDEFoRd R. D., BROOKE B. N., I966. A new-method of studying gastric, small intestinal, transit times. Gut, $7,7 \mp 8$ (Abstr.).

Rozen R., I967. Évacuation stomacale comparée du lait et du yoghourt chez le Rat. Ann. Biol.anim. Bioch. Biophys., 7, 47-58.

Ruckebusch Y., Grivel M.-L., Santini R., rg68. Technique de dérivation chronique de l'activité électrique et mécanique du tractus digestif chez l'animal éveillé. Bull. Soc. Sci. Vêt. Méd. Comp., 70, $337-345$.

Ruckebusch Y., Kay R. N. B., I97r. Étude critique de la motricité gastrique chez les bovins. Ann. Rech. vét. : 2, 99-1 36 .

Schedl H. P., Miller D., White D., rg66. Use of polyethylene glycol and phenol red as unabsorbed indicators for intestinal absorption studies in man. Gut, 7 , I59-163.

Schïrch A.F., Lloyd L. E., Crampton F. W., I96o. The use of chromic oxide as an index for determining the digestibility of a diet. J. Nutr., 41, 629-636.

Shields R., Harris J., Davies M. W., I 968 . Suitability of polyethylene glycol as a dilution indicator in the human colon. Gastroent., 54, 331-333.

Shtratori T., Okabayashi T., Harata N., Nagaoka K., ig69. Intraluminal lead of the gastric action potentials in human subjects : use of a new suction-needle electrode. Surgery, 66, 483-487.

Sikov M. R., Thomas J. M., Mahlum D. D., rg6g. Comparison of passage of a tracer through the gastro-intestinal tract of neo-natal and adult rats. Growth, 33, 57-68.

Sмiтн R. H., I958. Substances in the calf alimentary tract interfering in the determination of polyethyleneglycol. Nature, 182, 260-26r.

Smith R. H., 1959. The development and function of the rumen in milk fed calves. J. Agric. Sci., 52, $72-78$.

Smiti R. H., 1964. Passage of digesta through the calf abomasum and small intestine. J. Physiol., Londres, 172, 305-320. 
Smith A. N., Ridgway M., I962. The use of telemetering capsules in disorders of the alimentary tract. Part I : A technique for recording pressure changes. Gut. 3, 366-376.

Sperber I., Hyden S., Ekman J., r953. The use of polyethyleneglycol as a reference substance in the study of ruminant digestion. Ann. Roy. A gric. Coll. Sweden, 20, 337-344.

Spurrel W. R., 1935. Duodenal reflexes. J. Physiol., Londres, 84, 4-5.

Stordy S. N., Greig J. H., Bogoch A., I969. The steak and barium meal. A method for evaluating gastric emptying after partial gastrectomy. Amer. J. Dig. Dis., 14, 463-469.

Szurszewski J. H., Elveback L. R., CODE C. F., I97o. Configuration and frequency gradient of electric slow-wave over canine small bowel. Amer. $J$. Physiol., 218, r468-1473.

Thivend P., Poncet C., I97i. Prélèvement automatique de contenus intestinaux chez le Veau et le Mouton. Ann. Biol. anim. Bioch. Biophys., 11, 274-275.

Thomas J. E., Crider J. O., Morgan J., I934. A study of reflexes involving the pyloric sphincter and antrum and their role in gastric evacuation. Amer. J. Physiol., 108,683-70o,

Thouvenot J., Martin A., Touron P., ig68. Potentiels dérivés au niveau de la paroi abdominale: relation avec la motricité viscérale digestive chez l'Homme. J. Physiol., Paris, 60 (suppl. 2), 556-557.

TKAChEv E. Z., I969. Modification of the technique of application of double intestinal anastomosis in pigs (russe). Fiziologit., $2 h ., 60$, I $13-115$.

TodA N., ToBE T., I967. Studies on stereoroentgenography of motile organs. II. Gastro-intestinal tract.(japonais). Nippon Acta Radiol., 27, 391-405.

Tsuchida S., Kimura Y., I966. Electromyography of the intestines by the intra-intestinal method. Tohoku J. Exp. Med. Jap., 89, 61-68.

Werske H., I 897. Versuche über die Aufenthaltsdauer des Futters in Verdauungs apparate der Kaninchen. Landwirtsch. Versuchstationen, 48, 375-378.

Whitby L. G., LaNG D., I96o. Expérience with the chromic oxide method of fecal marking in metabolic balance investigation on humans. J. Clin. Invest., 39, 854-863.

Yamatani Y., Ishikawa S., I968. Polyvinyl alcohol as a water soluble marker. I. Absorption and excretion of polyvinyl alcohol from the gastro intestinal tract of adult rat. Agric. Biol. Chem., 32, $474-478$. 